\title{
A Solution for multi-evaluator AHP
}

\author{
Masaaki Shinohara \\ Nihon University \\ Izumi-chou, Narashino \\ Chiba 275-8575, Japan \\ M7sinoha@cit.nihon-u.ac.jp
}

\author{
Keikichi Osawa \\ Nihon University \\ Izumi-chou, Narashino \\ Chiba 275-8575, Japan \\ k7oosawa@cit.nihon-u.ac.jp
}

\author{
Yuko Hida \\ Nihon University \\ Izumi-chou, Narashino \\ Chiba 275-8575, Japan \\ m7sinoha@cit.nihon-u.ac.jp
}

Keywords: multi-evaluator, evaluator's weight, logarithmic least squares estimation

Summary: We often encounter the large scale AHP where there are many kinds of alternatives (or objects to be evaluated) and one evaluator cannot cover whole objects, so several separate evaluators are needed, further each evaluator has the specific ability to evaluate a specific group of objects. Let such type of AHP be called multi-evaluator AHP. To solve the multi-evaluator AHP, we propose a logarithmic linear pairwise comparison error model, taking evaluator's specific characteristic in consideration, and then, the least square principle is applied to obtain estimates of object weight and evaluator weight. The physical meaning of weights of evaluators is clarified by the specific formula obtained in our analysis. Further the so called group decision making in AHP is a special case of multi-evaluator AHP, by which we can evaluate the reliabilities of evaluators.

\section{Introduction}

We often encounter the large scale AHP[1] where there are many kinds of alternatives (or objects to be evaluated) and one evaluator cannot cover whole objects, so several separate evaluators are needed, further each evaluator has the specific ability to evaluate a specific group of objects. Let such type of AHP be called multi-evaluator AHP.

To solve the multi-evaluator AHP, we propose a logarithmic linear pairwise comparison error model, taking evaluator's specific characteristic in consideration, and then, the least square principle is applied to obtain estimates of object weight and evaluator weight.

Already K.Taji et al[3] proposed a solving method for this type of problem by ANP[2], which gives a well reasonable algorithm. Our approach is different from theirs and gives the following specific profits which are not seen in [3] ; The physical meaning of weights of evaluators is clarified by the specific formula obtained in our analysis. Further the so called group decision making [4],[5] in AHP is a special case of multi-evaluator AHP, by which we can evaluate the reliabilities of evaluators.

\section{The error model of multi-evaluator AHP}

Figure 1 shows a simple model of multi-evaluator AHP where there are 3 evaluators 
A,B,C and 5 objects $1,2, \cdots, 5$. A matches objects "1,2,3,", B matches objects "2,3,4," and C matches objects " $3,4,5$ ".

Each evaluator gives paired comparison values for the objects of his group like the ordinary AHP. Here let $a_{i j}\left(b_{i j}, c_{i j}\right)$ be the paired comparison value for objects $i$ and $j$ given by $\mathrm{A}(\mathrm{B}, \mathrm{C})$, and $u_{i}$ be the true value of object $i$. Each evaluator is considered to have different evaluating criterion, and let $\alpha(\beta, \gamma)$ be the weight of $\mathrm{A}(\mathrm{B}, \mathrm{C})$, for characterizing the evaluator's attitude, then we assume that

$$
\begin{aligned}
& a_{i j}^{\alpha} \approx\left(\frac{u_{i}}{u_{j}}\right)(i, j)=(1,2),(1,3),(2,3) \\
& b_{i j}^{\beta} \approx\left(\frac{u_{i}}{u_{j}}\right)(i, j)=(2,3),(2,4),(3,4) \\
& c_{i j}^{\gamma} \approx\left(\frac{u_{i}}{u_{j}}\right)(i, j)=(3,4),(3,5),(4,5) \\
&(“ \approx ” \text { means "approximation”). }
\end{aligned}
$$

This situation is also described by the graph shown in Figure2. Let it be called graph of multi - evaluator AHP.

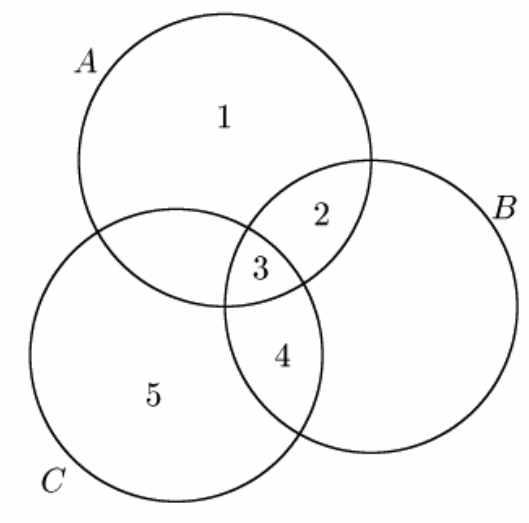

Figure 1: Structure of multi-evaluator AHP

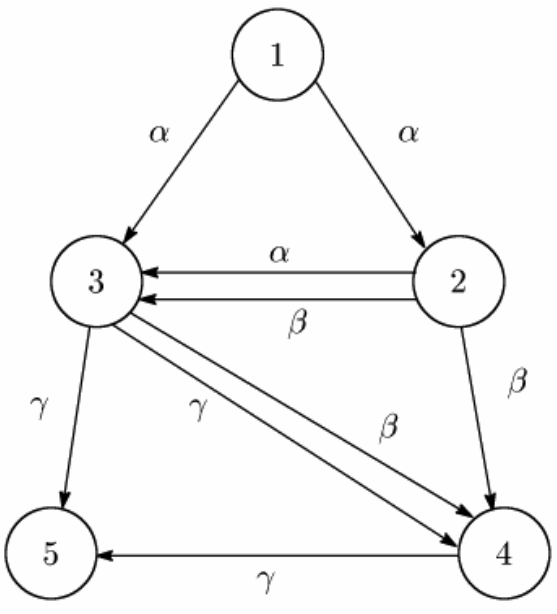

Figure 2: Graph of multi-evaluator AHP

Any connected graph with weighted directed arcs can be a graph of multi - evaluator AHP, as we see later examples. Note that $\operatorname{arc}(i, j)$ (origin $i$, end point $j$ ) with weight $\alpha$ corresponds to $a_{i j}^{\alpha}=\left(\frac{u_{i}}{u_{j}}\right)$ in the basic formula (1)

Taking logarithm of (1) we have

$$
\dot{a}_{i j} \alpha \approx \dot{u}_{i}-\dot{u}_{j}\left(\dot{b}_{i j} \beta \approx \dot{u}_{i}-\dot{u}_{j}, \dot{c}_{i j} \gamma \approx \dot{u}_{i}-\dot{u}_{j}\right)
$$


where $\dot{x}=\log _{e} x$ for $x=a_{i j}, u_{i}$, etc.

We apply the principle of least squares (LS) to (2) to make $L_{2}$-norm for the error in (2) minimal in $\alpha, \beta, \cdots, \dot{u}_{1}, \dot{u}_{2}, \cdots$.

$$
\mathrm{S}=\left\{\dot{a}_{12} \alpha-\left(\dot{u}_{1}-\dot{u}_{2}\right)\right\}^{2}+\left\{\dot{b}_{23} \beta-\left(\dot{u}_{2}-\dot{u}_{3}\right)\right\}^{2}+\cdots+\left\{\dot{c}_{45} \gamma-\left(\dot{u}_{4}-\dot{u}_{5}\right)\right\}^{2}
$$

The minimizing $\alpha(\beta, \gamma)$ is the estimate of the value of the weight of $A(B, C)$ and the minimizing $\dot{u}_{i}$ is the estimate of the true value of object $i \quad(i=1,2, \cdots, 5)$.

Here we assume the following constraints ;

$$
\begin{aligned}
& \dot{u}_{1}+\dot{u}_{2}+\dot{u}_{3}+\dot{u}_{4}+\dot{u}_{5}=0 \\
& \alpha+\beta+\gamma=3 \quad \text { The number of evaluators. })
\end{aligned}
$$

Generally $\dot{u}_{1}, \dot{u}_{2}, \cdots$ and $\dot{u}_{5}$ are free from a constant multiple so we can assume $u_{1} \cdot u_{2} \cdot u_{3} \cdot u_{4} \cdot u_{5}=1$, whose logarithm is (4). We can accept (5) because the standard values of evaluator weights are 1 .

\section{Solving method of multi-evaluator AHP}

Already we have described the solving principle in Chapter 2.

Here we formalize the principle by the new symbolism to solve general multi-evaluator AHP, and further describe several properties of the solution.

Firstly we introduce several symbols;

$1+\varepsilon_{v}$ :weight $\alpha_{v}$ of evaluator $v(v=1, \cdots, k) \quad\left(1+\varepsilon_{v}=\alpha_{v}\right)$

$\dot{u}_{i}:$ logarithm of the true value $u_{i}$ of object $i \quad(i=1, \cdots, m)$

$\dot{a}_{j}$ :logarithm of the paired comparison value of the evaluator corresponding to arc $j$ in the graph of multi-evaluator AHP $(j=1, \cdots, n)$.The order of the numbering of $j$ is the following; the set of arcs are decomposed into groups of evaluator $v \quad(v=1$, $\cdots, k)$, and arc $j=\left(i, i^{\prime}\right) \quad$ within group $v$ is specified by $\left(v, i, i^{\prime}\right)$, so the order of $j$ is determined lexicographically by $\left(v, i, i^{\prime}\right)$.

For example the graph in Figure 2 is rewritten by new symbols in Figure 


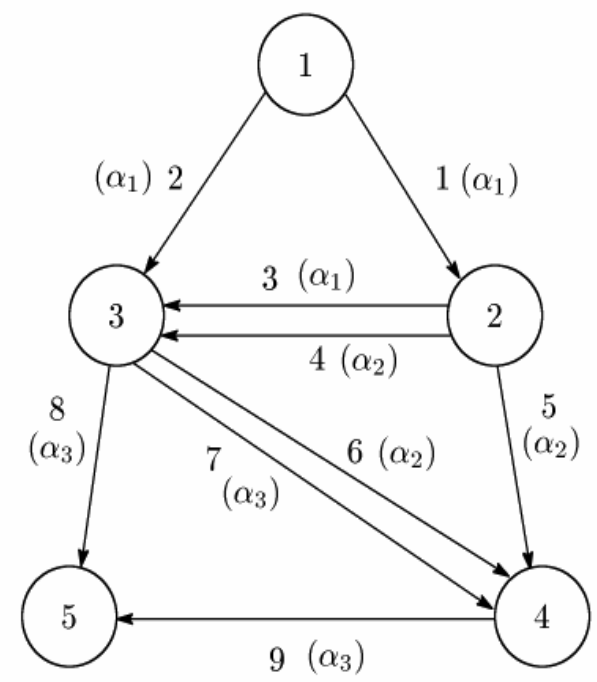

Figure 3: Graph of multi-evaluator AHP by new symbols $(\mathrm{k}=3, \mathrm{~m}=5, \mathrm{n}=9)$

Now the LS principle is to minimize

$$
S=\sum_{j=1}^{n}\left(\dot{a}_{j}\left(\varepsilon_{v}+1\right)-\left(\dot{u}_{i}-\dot{u}_{i^{\prime}}\right)\right)^{2}
$$

under the conditions (7) and (8), where $v, i, i^{\prime}$ is determined by $j$.

and

$$
\begin{gathered}
\dot{u}_{i}+\cdots+\dot{u}_{m}=0 \\
\varepsilon_{1}+\cdots+\varepsilon_{k}=0
\end{gathered}
$$

\section{Example 1}

We write down (6) (7) (8) for the multi - evaluator AHP shown in Figure 3.

$$
\begin{aligned}
& S=\left\{\left(\dot{a}_{1}\left(1+\varepsilon_{1}\right)-\left(\dot{u}_{1}-\dot{u}_{2}\right)\right)^{2}+\left(\dot{a}_{2}\left(1+\varepsilon_{1}\right)-\left(\dot{u}_{1}-\dot{u}_{3}\right)\right)^{2}\right\} \\
& +\left(\dot{a}_{3}\left(1+\varepsilon_{1}\right)-\left(\dot{u}_{2}-\dot{u}_{3}\right)\right)^{2}+\left(\dot{a}_{4}\left(1+\varepsilon_{2}\right)-\left(\dot{u}_{2}-\dot{u}_{3}\right)\right)^{2} \\
& +\left(\dot{a}_{5}\left(1+\varepsilon_{2}\right)-\left(\dot{u}_{2}-\dot{u}_{4}\right)\right)^{2}+\left(\dot{a}_{6}\left(1+\varepsilon_{2}\right)-\left(\dot{u}_{3}-\dot{u}_{4}\right)\right)^{2} \\
& +\left(\dot{a}_{7}\left(1+\varepsilon_{3}\right)-\left(\dot{u}_{3}-\dot{u}_{4}\right)\right)^{2}+\left(\dot{a}_{8}\left(1+\varepsilon_{3}\right)-\left(\dot{u}_{3}-\dot{u}_{5}\right)\right)^{2} \\
& \left.+\left(\dot{a}_{9}\left(1+\varepsilon_{3}\right)-\left(\dot{u}_{4}-\dot{u}_{5}\right)\right)^{2}\right\} \rightarrow \min \\
& \dot{u}_{1}+\dot{u}_{2}+\dot{u}_{3}+\dot{u}_{4}+\dot{u}_{5}=0 \\
& \varepsilon_{1}+\varepsilon_{2}+\varepsilon_{3}=0
\end{aligned}
$$

So the problem is an LS problem minimizing (6) with constraints (7) and (8). This can be solved by Lagrange method.

Let the Lagrange multipliers of (7) and (8) be $\lambda$ and $\mu$, respectively, and then 


$$
L=S+\lambda\left(\dot{u}_{1}+\cdots+\dot{u}_{m}\right)+\mu\left(\varepsilon_{1}+\cdots+\varepsilon_{k}\right) .
$$

Then the solution of our problem is obtained by solving the equations ,

$$
\begin{array}{ll}
\frac{\partial L}{\partial u_{i}}=0 & (i=1, \cdots, m) \\
\frac{\partial L}{\partial \varepsilon_{v}}=0 & (v=1, \cdots, k)
\end{array}
$$

with (7) and (8).

Taking (the root of) each term in (6) to be error $e_{j}$, we have

$$
\dot{a}_{j}=\dot{u}_{i}-\dot{u}_{i^{\prime}}-\dot{a}_{j} \varepsilon_{v}+e_{j} \quad(j=1, \cdots, n)
$$

which is represented by the matrix form

$$
a=C u-\left[\begin{array}{cccc}
a_{1} & 0 & \cdots & 0 \\
0 & a_{2} & \cdots & 0 \\
\vdots & \vdots & \ddots & \vdots \\
0 & 0 & \cdots & a_{k}
\end{array}\right] \varepsilon+e
$$

where

$$
a=\left[\begin{array}{l}
\dot{a}_{1} \\
\dot{a}_{2} \\
\vdots \\
\dot{a}_{n}
\end{array}\right]=\left[\begin{array}{l}
a_{1} \\
a_{2} \\
\vdots \\
a_{k}
\end{array}\right], u=\left[\begin{array}{l}
\dot{u}_{1} \\
\dot{u}_{2} \\
\vdots \\
\dot{u}_{m}
\end{array}\right], \quad \varepsilon=\left[\begin{array}{l}
\varepsilon_{1} \\
\varepsilon_{2} \\
\vdots \\
\varepsilon_{m}
\end{array}\right], \quad e=\left[\begin{array}{l}
e_{1} \\
e_{2} \\
\vdots \\
e_{m}
\end{array}\right]
$$

and $C$ is the incidence matrix of the graph of multi-evaluator AHP and $a_{v}$ is the vector whose components are paired comparison data by evaluator $v$ $(v=1, \cdots, k)$.

Table 1: Data Table

\begin{tabular}{|c|c|cccc|}
\multicolumn{1}{c}{} & $u_{1} \cdots, u_{m}$ & $\varepsilon_{1}$ & $\varepsilon_{2}$ & $\cdots$ & $\varepsilon_{k}$ \\
\hline \multirow{3}{*}{$a$} & \multirow{3}{*}{$C$} & $-a_{1}$ & & & \\
& & & $-a_{2}$ & & \\
& & & & $\ddots$ & $-a_{k}$ \\
\hline
\end{tabular}

(Data Table of Example 1) 


\begin{tabular}{|c|ccccc|ccc|}
\multicolumn{1}{c}{$\dot{u}_{1}$} & $\dot{u}_{2}$ & $\dot{u}_{3}$ & $\dot{u}_{4}$ & $\dot{u}_{5}$ & $\varepsilon_{1}$ & $\varepsilon_{2}$ & $\varepsilon_{3}$ \\
\hline$\dot{a}_{1}$ & 1 & -1 & 0 & 0 & 0 & $-\dot{a}_{1}$ & 0 & 0 \\
$\dot{a}_{2}$ & 1 & 0 & -1 & 0 & 0 & $-\dot{a}_{2}$ & 0 & 0 \\
$\dot{a}_{3}$ & 0 & 1 & -1 & 0 & 0 & $-\dot{a}_{3}$ & 0 & 0 \\
\hline$\dot{a}_{4}$ & 0 & 1 & -1 & 0 & 0 & 0 & $-\dot{a}_{4}$ & 0 \\
$\dot{a}_{5}$ & 0 & 1 & 0 & -1 & 0 & 0 & $-\dot{a}_{5}$ & 0 \\
$\dot{a}_{6}$ & 0 & 0 & 1 & -1 & 0 & 0 & $-\dot{a}_{6}$ & 0 \\
\hline$\dot{a}_{7}$ & 0 & 0 & 1 & -1 & 0 & 0 & 0 & $-\dot{a}_{7}$ \\
$\dot{a}_{8}$ & 0 & 0 & 1 & 0 & -1 & 0 & 0 & $-\dot{a}_{8}$ \\
$\dot{a}_{9}$ & 0 & 0 & 0 & 1 & -1 & 0 & 0 & $-\dot{a}_{9}$ \\
\hline
\end{tabular}

Formula (12) (with omitting error term) is often represented by a table called data table shown in Table 1.

Let the coefficient matrix of Data Table to be $X$,

$$
X=\left[\begin{array}{l|lll} 
& C & -a_{1} & \\
& \ddots & \\
& & & -a_{k}
\end{array}\right]
$$

then the normal equation of this LS problem is given by (15), where $e_{m}$ and $e_{k}$ are all 1 column vectors of dimension $m$ and $k$, respectively

$$
M\left[\begin{array}{c}
u \\
\varepsilon \\
\lambda \\
\mu
\end{array}\right]=\left[\begin{array}{c}
X^{T} a \\
0 \\
0
\end{array}\right], \quad M=\left[\begin{array}{cccc}
X^{T} X & & e_{m} & 0 \\
& & 0 & e_{k} \\
e_{m}^{T} & 0 & 0 & 0 \\
0 & e_{k}^{T} & 0 & 0
\end{array}\right]
$$

And the solutions of (15) 


$$
u=\left[\hat{\dot{u}}_{1, \ldots}, \hat{\dot{u}}_{m}\right]^{T}, \hat{\varepsilon}=\left[\hat{\varepsilon}_{1, \cdots}, \hat{\varepsilon}_{k}\right]^{T}, \hat{\lambda}, \hat{\mu}
$$

are our desired results.

\section{Example 2}

Consider a multi-evaluator AHP shown in Figure 4.

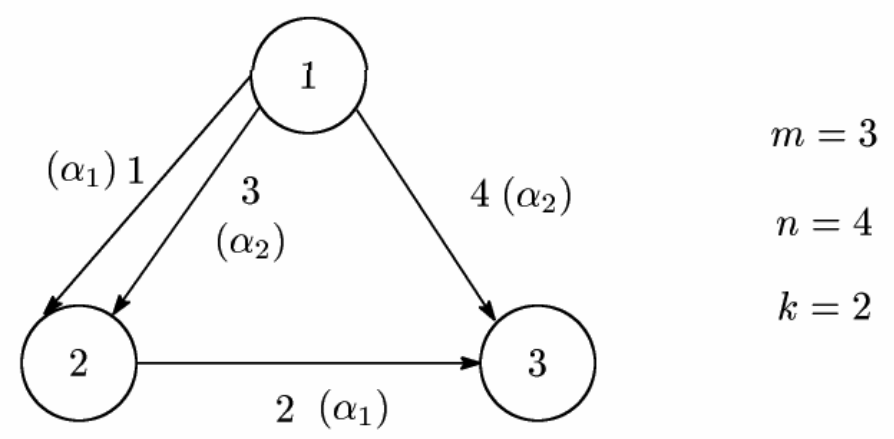

Figure 4: Simple example of multi-evaluator AHP

For this example, we have

$$
X=\left[\begin{array}{ccccc}
1 & -1 & 0 & -\dot{a}_{1} & 0 \\
0 & 1 & -1 & -\dot{a}_{2} & 0 \\
1 & -1 & 0 & 0 & -\dot{a}_{3} \\
1 & 0 & -1 & 0 & -\dot{a}_{4}
\end{array}\right],
$$

and the normal equation of (15) is shown in Table 2.

\begin{tabular}{|c|c|c|c|c|c|c|c|}
\hline$\dot{u}_{1}$ & $\dot{u}_{2}$ & $\dot{u}_{3}$ & $\varepsilon_{1}$ & $\varepsilon_{2}$ & $\lambda$ & $\mu$ & \\
\hline 3 & -2 & -1 & $-\dot{a}_{1}$ & $-\dot{a}_{3}-\dot{a}_{4}$ & 1 & 0 & $\dot{a}_{1}+\dot{a}_{3}+\dot{a}_{4}$ \\
\hline-2 & 3 & -1 & $\dot{a}_{1}-\dot{a}_{2}$ & $\dot{a}_{3}$ & 1 & 0 & $-\dot{a}_{1}+\dot{a}_{2}-\dot{a}_{3}$ \\
\hline-1 & -1 & 2 & $\dot{a}_{2}$ & $\dot{a}_{4}$ & 1 & 0 & $-\dot{a}_{2}-\dot{a}_{4}$ \\
\hline$-\dot{a}_{1}$ & $\dot{a}_{1}-\dot{a}_{2}$ & $\dot{a}_{2}$ & $\dot{a}_{1}^{2}+\dot{a}_{2}^{2}$ & 0 & 0 & 1 & $-\dot{a}_{1}^{2}-\dot{a}_{2}^{2}$ \\
\hline$-\dot{a}_{3}-\dot{a}_{4}$ & $\dot{a}_{3}$ & $\dot{a}_{4}$ & 0 & $\dot{a}_{3}^{2}+\dot{a}_{4}^{2}$ & 0 & 1 & $-\dot{a}_{3}^{2}-\dot{a}_{4}^{2}$ \\
\hline 1 & 1 & 1 & 0 & 0 & 0 & 0 & 0 \\
\hline 0 & 0 & 0 & 1 & 1 & 0 & 0 & 0 \\
\hline
\end{tabular}

Table 2: Normal equation of multi-evaluator AHP

For $a_{1}=1\left(\dot{a}_{1}=0\right), a_{2}=3\left(\dot{a}_{2}=1.099\right), a_{3}=4\left(\dot{a}_{3}=1.386\right), a_{4}=2\left(\dot{a}_{4}=0.693\right)$, 
the solution is given as below

$\hat{\dot{u}}_{1}=0.40672, \hat{\dot{u}}_{2}=0.235366, \hat{\dot{u}}_{3}=-0.64209$,

$\hat{\varepsilon}_{1}=0.330681\left(\hat{\alpha}_{1}=0.751\right), \hat{\varepsilon}_{2}=-0.330681\left(\hat{\alpha}_{2}=1.494\right)$,

$\hat{u}_{1}=1.501883, \hat{u}_{2}=1.265371, \hat{u}_{3}=0.526191, \hat{\lambda}=0, \hat{\mu}=-0.64288$.

\section{Physical meaning of evaluators weight}

Decomposing (15) into u-part and $\varepsilon$-part by (14), we have

$$
\begin{gathered}
C^{\mathrm{T}} \mathrm{Cu}-\left(\mathrm{C}_{1}^{\mathrm{T}} a_{1} \hat{\varepsilon}_{1}+\cdots+\mathrm{C}_{\mathrm{k}}^{\mathrm{T}} a_{\mathrm{k}} \hat{\varepsilon}_{k}\right)+\hat{\lambda} e_{m}=C^{T} a \\
\text {,and } \quad-a_{v}^{\mathrm{T}} C_{v} \hat{\mathrm{u}}+\left(a_{v}^{\mathrm{T}} a_{v}\right) \hat{\varepsilon}_{v}+\hat{\mu}=-\left(a_{v}^{\mathrm{T}} a_{v}\right), \quad v=1, \cdots, k
\end{gathered}
$$

Here $C_{v}$ is $v$-th decomposed part (corresponding to evaluator $v$ ) of $C$, that is

$$
C=\left[\begin{array}{c}
C_{1} \\
C_{2} \\
\vdots \\
C_{k}
\end{array}\right] .
$$

By (17) and (18) we have the following theorems .

Theorem 1 The LS solution $\hat{\lambda}$ of Lagrange multiplier $\lambda$ for (7) is always zero. Proof It is clear that

$$
C e_{m}=\left[\begin{array}{c}
C_{1} e_{m} \\
\vdots \\
C_{k} e_{m}
\end{array}\right]=0 .
$$

because $C$ is the incidence matrix of the graph of multi-evaluator AHP.

So we have also $e_{m}^{T} C^{T}=0$. Multiplying (17) from the left by $e_{m}^{T}$ we have

$$
\hat{\lambda} e_{m}^{T} e_{m}=0
$$

which shows $\hat{\lambda}=0$.

Theorem 2 The LS solution $\hat{\mu}$ of Lagrange multiplier $\mu$ for (8) is

$$
\hat{\mu}=\left(\sum_{v} \frac{a_{v}^{T} C_{v} \hat{\mathrm{u}}}{a_{v}^{T} a_{v}}-k\right) / \sum_{v} \frac{1}{a_{v}^{T} a_{v}} .
$$

Proof Dividing (18) by $a_{v}^{T} a_{v}$ and summing up on $v=1, \cdots, k$ we have by (8) 


$$
-\sum_{v} \frac{a_{v}^{T} C_{v} \hat{\mathrm{u}}}{a_{v}^{T} a_{v}}+\hat{\mu} \sum_{v} \frac{1}{a_{v}^{T} a_{v}}=-k
$$

which leads us to $(20)$.

Translating $\hat{\varepsilon}_{v}=\hat{\alpha}_{v}-1$ to $\hat{\alpha}_{v}$, we have by (18)

$$
\hat{\alpha}_{v}=\frac{a_{v}^{T} C_{v} \hat{\mathrm{u}}-\hat{\mu}}{a_{v}^{T} a_{v}} \quad v=1, \cdots, k .
$$

Formula (21) has very important physical meaning of evaluator $v$, which we explain through the following example.

Example 3 Consider the multi-evaluator AHP in Figure 3.

The decomposition of $C$ is given as below.

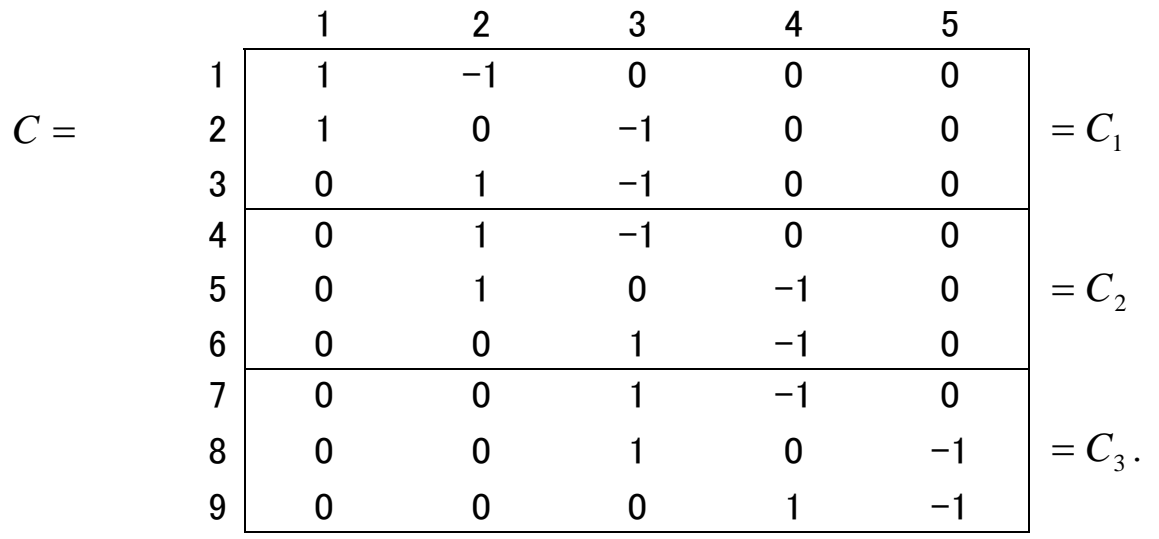

$$
\begin{aligned}
& \hat{\alpha}_{1}=\left\{\dot{a}_{1}\left(\hat{\dot{u}}_{1}-\hat{\dot{u}}_{2}\right)-\dot{a}_{2}\left(\hat{\dot{u}}_{1}-\hat{\dot{u}}_{3}\right)+\dot{a}_{3}\left(\hat{\dot{u}}_{2}-\hat{\dot{u}}_{3}-\hat{\mu}\right)\right\} /\left(\dot{a}_{1}^{2}+\dot{a}_{2}^{2}+\dot{a}_{3}^{2}\right) \\
& \hat{\alpha}_{2}=\left\{\dot{a}_{4}\left(\hat{\dot{u}}_{2}-\hat{\dot{u}}_{3}\right)-\dot{a}_{5}\left(\hat{\dot{u}}_{2}-\hat{\dot{u}}_{4}\right)+\dot{a}_{6}\left(\hat{\dot{u}}_{3}-\hat{\dot{u}}_{4}-\hat{\mu}\right)\right\} /\left(\dot{a}_{4}{ }^{2}+\dot{a}_{5}{ }^{2}+\dot{a}_{6}{ }^{2}\right) \\
& \hat{\alpha}_{3}=\left\{\dot{a}_{7}\left(\hat{\dot{u}}_{3}-\hat{\dot{u}}_{4}\right)-\dot{a}_{8}\left(\hat{\dot{u}}_{3}-\hat{\dot{u}}_{5}\right)+\dot{a}_{9}\left(\hat{\dot{u}}_{3}-\hat{\dot{u}}_{5}-\hat{\mu}\right)\right\} /\left(\dot{a}_{7}{ }^{2}+\dot{a}_{8}{ }^{2}+\dot{a}_{9}{ }^{2}\right)
\end{aligned}
$$

If the comparison data of evaluators are all exact, that is

, then generally

$$
\begin{gathered}
\dot{a}_{1}=\hat{\dot{u}}_{1}-\hat{\dot{u}}_{2} \\
\dot{a}_{2}=\hat{\dot{u}}_{1}-\hat{\dot{u}}_{3} \\
\dot{a}_{3}=\hat{\dot{u}}_{2}-\hat{\dot{u}}_{3} \\
\quad \vdots \\
\dot{a}_{9}=\hat{\dot{u}}_{3}-\hat{\dot{u}}_{5}
\end{gathered}
$$

$$
\frac{a_{v}^{T} C_{v} \hat{\mathrm{u}}}{a_{v}^{T} a_{v}}=1 \quad v=1, \cdots, k
$$

Then, by Theorem 2, we have $\hat{\mu}=0$. We can say that $\hat{\mu}$ is a kind of general criterion to measure the accuracy of evaluators. 
When $\hat{\mu}=0, \hat{\alpha}_{v}=a_{v}^{T} C_{v} \hat{\mathrm{u}} / a_{v}^{T} a_{v}$ is a kind of correlation coefficient of comparison data $\dot{a}_{j}$ and corresponding $\dot{u}_{i}-\dot{u}_{i^{\prime}}$ in evaluator $v$. So the evaluator having $\hat{\alpha}_{v}$ near to 1 is reliable evaluator.

If evaluator $v$ has a tendency of underestimates for paired comparisons

$$
\delta_{v}=a_{v}^{T} C_{v} \hat{\mathrm{u}}-a_{v}^{T} a_{v}
$$

becomes positive, so

$$
\hat{\alpha}_{v}=a_{v}^{T} C_{v} \hat{\mathrm{u}} / a_{v}^{T} a_{v}=1+\delta_{v} / a_{v}{ }^{T} a_{v}
$$

becomes larger than 1 . The contrary case is also valid, and as a results we can say;

evaluator $v$ having $\hat{\alpha}_{v} \fallingdotseq 1$ is reliable one ,and having $\hat{\alpha}_{v}>1(<1)$ has a tendency of under(over)estimates for paired comparisons. And further by Theorem 2 we can say;

If $\hat{\mu} \fallingdotseq 0$, it shows that as a whole evaluators are reliable and $\hat{\mu}>0(<0)$ shows that evaluators as a whole have a tendency of under(over)estimating.

The so called "group decision problem"(GDP) in AHP is a special case of multi-evaluator AHP. GDP treats the problem where every evaluator covers all objects. A simple case of GDP $(\mathrm{m}=3, \mathrm{k}=2)$ is shown in Fig. 5 .

For the analysis of GDP, we have only to take $C_{v}(v=1, \cdots, k)$ equal to $C$ (incidence matrix of the graph of multi-evaluator AHP) in (18) and (19).

For example the data table for Fig. 5 is shown in Table 3. We can see the duplicates of $C$ in the column of $\mathbf{u}$-part.

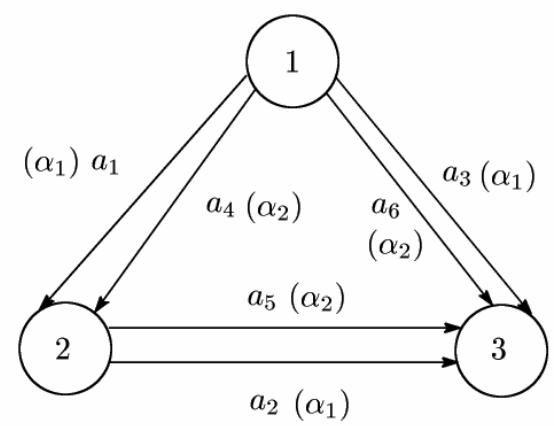

Fig 5. Simple GDP Example

\begin{tabular}{|c|c|c|c|c|c|}
\hline & $\dot{u}_{1}$ & $\dot{u}_{2}$ & $\dot{u}_{3}$ & $\varepsilon_{1}$ & $\varepsilon_{2}$ \\
\hline$\dot{a}_{1}$ & 1 & -1 & 0 & $-\dot{a}_{1}$ & 0 \\
\hline$\dot{a}_{2}$ & 1 & 0 & -1 & $-\dot{a}_{2}$ & 0 \\
\hline$\dot{a}_{3}$ & 0 & 1 & -1 & $-\dot{a}_{3}$ & 0 \\
\hline$\dot{a}_{4}$ & 1 & -1 & 0 & 0 & $-\dot{a}_{4}$ \\
\hline
\end{tabular}

Table 3: Data Table of GDP Example 


\begin{tabular}{c|ccc|cc}
$\dot{a}_{5}$ & 1 & 0 & -1 & 0 & $-\dot{a}_{5}$ \\
$\dot{a}_{6}$ & 0 & 1 & -1 & 0 & $-\dot{a}_{6}$ \\
\hline 0 & 1 & 1 & 1 & 0 & 0 \\
0 & 0 & 0 & 0 & 1 & 1 \\
\hline
\end{tabular}

Conventional analysis of GDP is to take the geometric mean of the results of evaluators, but by this conventional analysis we cannot see the evaluators abilities. However we can clarify the evaluators weight $\alpha_{v}$ by the above statements.

For

$a_{1}=1.1\left(\dot{a}_{1}=0.095\right), a_{2}=2.2\left(\dot{a}_{2}=0.788\right), a_{3}=2.5\left(\dot{a}_{3}=0.916\right), a_{4}=0.9\left(\dot{a}_{4}=-0.105\right)$, $a_{5}=2.0\left(\dot{a}_{5}=0.693\right)$ and $a_{6}=2.4\left(\dot{a}_{6}=0.875\right)$ the solutions is given as below

$$
\begin{array}{lrlrl}
\hat{\dot{u}}_{1}=0.243, & \hat{\dot{u}}_{2}=0.301, & \hat{\dot{u}}_{3}=-0.545, & \\
& & & \\
\hat{\varepsilon}_{1}=-0.042 & \left(\hat{\alpha}_{1}=0.751\right), & \hat{\varepsilon}_{2}=0.042 & \left(\hat{\alpha}_{2}=1.494\right), & \\
\hat{u}_{1}=1.275 & \hat{u}_{2}=1.351 & \hat{u}_{3}=0.580 & \hat{\lambda}=0 & \hat{\mu}=-0.018
\end{array}
$$

\section{Conclusion and further research}

We proposed a solving method of multi-evaluator AHP based on the constrained least squares (LS) method, which gives not only the estimates of weights of alternatives but also those of evaluators (Chapter 3).

Further by our method the physical meanings of evaluator's weight is clarified through Lagrange multiplier for the constraint. We can solve the group decision problems in AHP by our method as a special case of multi-evaluator AHP (Chapter 4).

We would like to extend multi-evaluator AHP to design problems which inform each evaluator to select what subset of whole objects to evaluate.

\section{References}

[1] T.L.Saaty :The Analytic Hierarchy Process(Mc-Graw Hill)(1980)

[2] T.L.Saaty :The Analytic Network Process(RWS-Publication)(1996)

[3]K.Taji, Y.Sagayama and H.Tamura :A Group Analytic Network Process(ANP) for Incomplete informatic, System Seigyo Joho

Gakkai,40(2002)305-311(in Japanese)

[4]M.Nakanishi and E.Kinoshita :An Application of the Group Decision Making Stress Method to the Group Analytic Hierarchy Process, Journal of The Operations Research Society of Japan,41(1998)560-570(in Japanese)

[5]Yoshiyasu Yamada. Manabu Sugiyama and Naokazu Yamaki, Group Analytic Hierarchy Process Based on Consensus Making Model, Journal of The Operations Research Society of Japan, 40 (1997) 236 244 (in Japanese) 